\title{
Up-Regulation of MiR-1915 Inhibits Proliferation, Invasion, and Migration of Helicobacter pylori-Infected Gastric Cancer Cells via Targeting RAGE
}

\author{
Xin-cai Xu, Wen-bin Zhang, Chun-xing Li, Hua Gao, Qi Pei, Bo-wei Cao, and Tie-han He \\ Department of Gastrointestinal Tumor, The First Affiliated Hospital of Xinjiang Medical University, Urumqi, China.
}

\begin{abstract}
Purpose: Helicobacter pylori (HP)-infected gastric cancer (GC) is known to be a fatal malignant tumor, but the molecular mechanisms underlying its proliferation, invasion, and migration remain far from being completely understood. Our aim in this study was to explore miR-1915 expression and its molecular mechanisms in regulating proliferation, invasion, and migration of $H P$-infected GC cells.

Materials and Methods: Quantitative real-time PCR and western blot analysis were performed to determine miR-1915 and receptor for advanced glycation end product (RAGE) expression in HP-infected GC tissues and gastritis tissues, as well as human gastric mucosal cell line GES-1 and human GC cell lines SGC-7901 and MKN45. CCK8 assay and transwell assay were performed to detect the proliferation, invasion, and migration capabilities. MiR-1915 mimics and miR-1915 inhibitor were transfected into GC cells to determine the target relationship between miR-1915 and RAGE.

Results: MiR-1915 was under-expressed, while RAGE was over-expressed in HP-infected GC tissues and GC cells. Over-expressed miR-1915 could attenuate cellular proliferation, invasion, and migration capacities. RAGE was confirmed to be the target gene of miR-1915 by bioinformatics analysis and luciferase reporter assay. Moreover, $H P$-infected GC cellular proliferation, invasion, and migration were inhibited after treatment with pcDNA-RAGE.

Conclusion: MiR-1915 exerted tumor-suppressive effects on cellular proliferation, invasion, and migration of $H P$-infected GC cells via targeting RAGE, which provided an innovative target candidate for treatment of $H P$-infected GC.
\end{abstract}

Key Words: Gastric cancer, microRNA-1915, MKN45, receptor for advanced glycation end product, SGC-7901

\section{INTRODUCTION}

Gastric cancer (GC) is becoming more common all around the world. In fact, it is believed to be the third most common of all cancers among males, as well as the fifth most common among females. ${ }^{1}$ Lower than $20 \%$ of GC patients can survive

Received: August 20, 2018 Revised: November 5, 2018

Accepted: November 20, 2018

Corresponding author: Wen-bin Zhang, MD, Department of Gastrointestinal Tumor, The First Affiliated Hospital of Xinjiang Medical University, 137 Liyushan South Rd, Urumqi, Xinjiang Uygur Autonomous Region, 830000, China.

Tel: 86-0991-4366260, Fax: 86-0991-4366260, E-mail: zwb3216@sina.com

-The authors have no potential conflicts of interest to disclose.

(C) Copyright: Yonsei University College of Medicine 2019

This is an Open Access article distributed under the terms of the Creative Commons Attribution Non-Commercial License (https://creativecommons.org/licenses/ by-nc/4.0) which permits unrestricted non-commercial use, distribution, and reproduction in any medium, provided the original work is properly cited. for more than 5 years after diagnosis. The incidence of GC has reduced in developed countries, whereas it is increasing in developing countries. ${ }^{2}$ Helicobacter pylori (HP) is a gram-negative bacterium that plays an important role in the pathogenesis of GC. ${ }^{3}$ About $60 \%$ of GC patients in developed countries and $75 \%$ in developing countries have chronic infection of $H P .{ }^{4}$ Chronic gastritis caused by $H P$ infection leads to the release of pathogenic factors like urease, vacuolating toxin A, CagA protein, inflammatory mediator, and reactive oxygen metabolites, causing aberrant hyperplasia and apoptosis of gastric mucosal epithelial cells, eventually leading to GC. ${ }^{5,6}$ Despite recent advances in treatments, including surgery, chemotherapy, and radiation therapy, GC still remains a difficult cancer to cure. ${ }^{7}$ Therefore, to cure GC, certain molecular mechanisms underlying GC cells proliferation, invasion, and migration, especially in $H P$-infected GC must be elucidated.

MicroRNAs (miRNAs) are endogenous noncoding RNAs with 
17-25 nucleotides, which play considerable roles in gene regulation of pathogenesis. ${ }^{8}$ Recent studies have confirmed that miR-1915 is related to several types of cancer, such as hepatocellular cancer, ${ }^{9}$ lung cancer, ${ }^{10}$ breast cancer, ${ }^{11}$ and colorectal cancer. ${ }^{12}$ Importantly, miR-1915 was down-regulated in GC cells, ${ }^{13}$ which might be major miRNA targets deserving further investigation. Nevertheless, the molecular mechanisms of miR1915 trigger $H P$-infected GC remain elusive.

Receptor for advanced glycation end product (RAGE) is a member of the immunoglobulin superfamily, which consists of more than 400 amino acids with a molecular weight of 35 $\mathrm{kD}$. RAGE has been shown to be linked with poor prognosis in GC patients, ${ }^{14}$ and our early study has also proven that knockdown of RAGE inhibited growth and invasion of GC cells. ${ }^{15}$ In addition, RAGE is over-expressed in GC tissues, especially in $H P$-infected GC cells. ${ }^{16}$ These data indicated that RAGE may be strongly linked to the pathogenesis of $H P$-infected GC, although the mechanisms behind disease progression remain unknown. Notably, we found that miR-1915 and RAGE sequences have binding sites by using bioinformatics software (microRNA.org), which demonstrated that RAGE may be a downstream target molecule of miR-1915.

In this context, we tried to clarify whether miR-1915 is causally involved in GC with HP infection, by performing several systematic and bioinformatic approaches and studying GC cell lines and GC tissues from human stomach biopsy specimens. Furthermore, our work aims to define the molecular mechanisms between miR-1915 and RAGE in regulation of cell proliferation, invasion, and migration of $H P$-infected GC cells.

\section{MATERIALS AND METHODS}

\section{Human tissue specimens}

This study on human beings has been approved by the Institutional Review Board (IRB) of The First Affiliated Hospital of Xinjiang Medical University. For the role of materials for research purposes, written informed consent was received from each patient. Human tissue specimens were accumulated and classified as described. ${ }^{17}$ Twenty pairs of $H$. pylori-positive gastritis $[H P(+)$ Gastritis] and negative gastritis $[H P(-)$ Gastritis] tissues were obtained from patients who underwent gastroscrope in The First Affiliated Hospital of Xinjiang Medical University. Thirty GC tissues were obtained randomly from patients who underwent gastrectomy in the same hospital. All GC tissues were identified by rapid urease test, and positive specimens were assigned to $H$. pylori-positive GC $[H P(+) \mathrm{GC}]$ group, while negative ones were assigned to $H$. pylori-negative GC $[H P(-) \mathrm{GC}]$ group. There were 16 specimens in $H P(+)$ GC group and 14 specimens in $H P(-)$ GC group. All tissue specimens were formaldehyde-fixed paraffin-embedded.

\section{Cell lines, $H$. pylori strain and $H P$ infection}

Human normal gastric epithelial cell line (GES-1) was bought from Shanghai Institute of Cell Biology (Shanghai, China). Human GC cell lines (SGC-7901 and MKN45) and wild-type $H$. pylori strain 26695 were bought from American Type Culture Collection (ATCC, Manassas, VA, USA). Infection procedure was performed as described. ${ }^{18}$ Cell lines SGC-7901 and MKN45 were propagated in DMEM (Gibco, Invitrogen, Waltham, MA, USA) with $10 \% \mathrm{FBS}$ in a humidified incubator $\left(5 \% \mathrm{CO}_{2}\right.$ at $\left.37^{\circ} \mathrm{C}\right)$. After cells grew to be approximately $80 \%$ confluent, they were co-cultured with HP at multiplicity of infection (M.O.I) of 100:1. After $12 \mathrm{~h}$ of infection, total RNA was extracted. All cell lines were maintained in RPMI 1640 that was supplemented in a humidified atmosphere of $95 \%$ air and $5 \% \mathrm{CO}_{2}$ with $10 \%$ fetal calf serum at $37^{\circ} \mathrm{C}$, which were identified by authentication.

\section{Quantitative real-time PCR}

Total RNAs were extracted from gastric tissues or cell lines using TRIZOL reagent (Invitrogen, Carlsbad, CA, USA). Reverse Transcription Kit (Takara, Dalian, China) was used to reverse transcribe from RNA to cDNA. The expressions of miRNAs and mRNAs were analyzed by quantitative real-time PCR (qRTPCR) using Power SYBR Green (Takara) with U6 or GAPDH as endogenous controls, respectively. For detection of miR-1915 expression, reverse transcription was performed as Applied Biosystems TaqMan MicroRNA Assay (Foster City, CA, USA) protocol. The RNA sequences used in this study were as follows: miR-1915: (Forward) 5'-CCCAAGCTTGGAAATCCGAC CACTA-3', (Reverse) 5'-CATGCCATGGCAGGATAGCAGC AC-3'; RAGE: (Forward) 5'-GTGTCCTTCCCAACGGCTC-3', (Reverse) 5'-ATTGCCTGGCACCGGAAAA-3'; $\beta$-actin: (Forward) 5'-TGATCCACATCTGCTGGAAGGT-3', (Reverse) 5'-GA CAGGATGCAGAAGGAGATTACT-3'. Relative expression levels of all genes were calculated as $2^{-\Delta \Delta \mathrm{Ct}}$.

\section{Western blot analysis and antibodies}

Total proteins were extracted from specimens or cell lines by $10 \%$ SDS-poly acrylamide gel electrophoresis, and transferred to poly-vinylidene difluoride membranes (Millipore, Burlington, MA, USA). After blocking with 5\% fat-free milk in 1×TBST for $1 \mathrm{~h}$, total proteins were immunoblotted with specific antibodies at $4^{\circ} \mathrm{C}$ overnight. After three times of washing, the proteins were incubated with horseradish peroxidase-conjugated secondary antibodies for $1 \mathrm{~h}$ at room temperature. After another three times of washing, protein bands were detected by SmartChemi (Beijing Sage Creation Science Co, Beijing, China). Antibody $\beta$-actin was used as internal reference.

\section{Cell transfection}

The 293T cells were bought from Shanghai Institute of Cell Biology. The cells $\left(2 \times 10^{4}\right.$ cells/well $)$ were cultured in 24 -well plates overnight, and transiently transfected using transfection reagent lipofectamine 2000 (Invitrogen). MiR-1915 mimic and 
negative control mimics (pre-NC), miR-1915 inhibitor, and negative control inhibitor (NC) were purchased from RiboBio Co., Ltd. (Guangzhou, China).

\section{Cell counting kit-8 (CCK-8) assay}

The 7901 or MKN45 cells $\left(2 \times 10^{3}\right.$ cells/well) were cultured in 96 -well plates in $100-\mu \mathrm{L}$ culture medium overnight. CCK-8 reagent ( $10 \mu \mathrm{L} /$ well) was added, and they were incubated for another $4 \mathrm{~h}$ with $5 \% \mathrm{CO}_{2}$ at $37^{\circ} \mathrm{C}$. We observed and recorded the procedures of cell growth at $0,24,48$, and $72 \mathrm{~h}$. Cell viability was determined at $450 \mathrm{NM}$, and expressed as a percentage of the control using a microplate reader (Bio-Rad, Hercules, CA, USA).

\section{Migration and invasion assay}

Capability of cell migration was examined by transwell migration assays. Transwell migration chambers were performed. Cells were seeded to $1 \times 10^{5}$ cells in serum-free media in the upper chamber with noncoated membrane $(8 \mu \mathrm{m}$ in pore size; Millipore, Schaffhausen, Switzerland). Lower chamber contained media with $20 \%$ FBS as a chemoattractant. Cells in upper chamber were discarded by using cotton wool after $24 \mathrm{~h}$, and migration cells in lower chamber were counted by microscope. For invasion assay, the experiments were the same as transwell migration assays, except that cells grew to $2 \times 10^{5}$ cells in upper chamber, which was coated with Matrigel (Sigma, St. Louis, MO, USA).

\section{Luciferase reporter assay}

We used TargetScan Human Release 6.2 (http://www.targetscan. org) to determine predicted target genes as well as their binding sites. The 3'-UTR sequence of RAGE was a candidate target gene of miR-1915, and was inserted into the pmirGLO Dual-Luciferase miRNA Target Expression Vector. The 293T cells were plated in 24-well plates and co-transfected with the vec- tor carrying wild-type (WT) or mutated (Mut) RAGE 3'-UTR and miR-1915 mimic or the negative control (NC). After $48 \mathrm{~h}$ of transfection, luciferase activity was detected by luciferase reporter assay system (Promega, Madison, WI, USA).

\section{Statistical analysis}

SPSS version 18.0 (SPSS Inc, Chicago, IL, USA) was used for statistical analysis, with either a Student's t-test or analysis of variance. All experiments were performed in triplicate, and data were presented as mean \pm standard deviation. $p$-values less than 0.05 were considered statistically significant.

\section{RESULTS}

Lowly-expressed miR-1915 and over-expressed RAGE are detected in $H P(+)$ GC tissues in vivo

MiRNAs and RAGE have been shown to be causally involved in gastric malignancy. ${ }^{13,15}$ We assumed that cell proliferation, invasion, and migration of $H P(+)$ GC cells were promoted by molecular regulation between them. We first examined miRNA expression levels in $H P$-infected and uninfected GC tissues. We gathered 20 pairs of gastritis specimens as control, and 30 GC specimens with $16 H P(+)$ and $14 H P(-)$ specimens. Several miRNAs, such as miR-20b, ${ }^{19}$ miR-342, ${ }^{20}$ miR-577, ${ }^{21}$ miR-767-5p, ${ }^{22}$ and miR-1915, ${ }^{13}$ were believed to participate in gastric tumorigenesis, and their expressions in GC tissues were detected by qRT-PCR. Only the expression of miR-1915 was significantly reduced in $H P(+)$ or $H P(-)$ GC groups compared to the control groups, while other miRNAs expressions were increased (Supplementary Fig. 1, only online). Among them, miR-1915 was found to be an intriguing candidate, as it played an important role in GC (significant lowly-expressed in GC tissues).

To determine the expression of miR-1915 and RAGE during the development of $\mathrm{HP}$-associated GC in vivo, we further

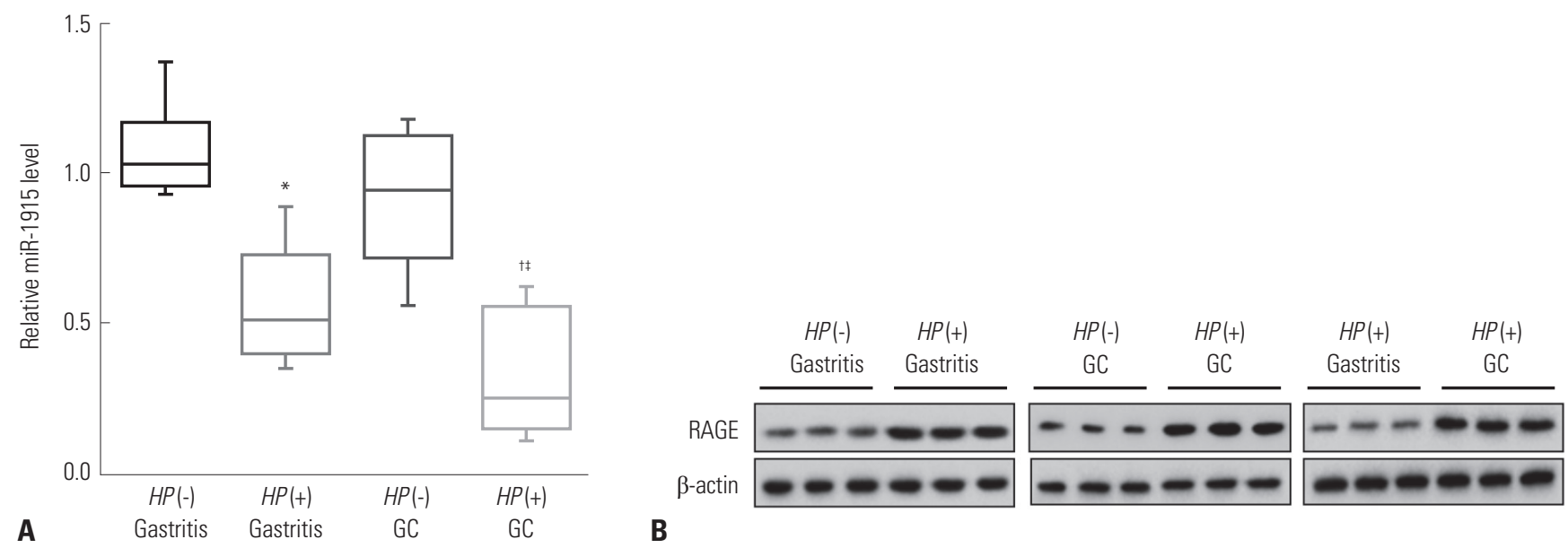

Fig. 1. Under-expressed miR-1915 and over-expressed RAGE in $H P(+) \mathrm{GC}$ tissues. (A) Relative miR-1915 level in gastritis or GC tissues with or without $H P$ infection by qRT-PCR. (B) Expression of RAGE in gastritis or GC tissues with or without $H P$ infection by western blotting. ${ }^{*} p<0.05$ vs. $H P(-)$ Gastritis, ${ }^{\dagger} p<0.05$ vs. $H P(-) G C,{ }^{\ddagger} p<0.05$ vs. $H P(+)$ Gastritis. RAGE, receptor for advanced glycation end product; $H P$, Helicobacter pylori, GC, gastric cancer. 
studied gastric tissues from patients with or without $H P$ infection. The qRT-PCR results showed that the expression of miR1915 was down-regulated in $H P(+)$ Gastritis and GC groups compared to $H P(-)$ groups. Additionally, miR-1915 expression remained under-expressed in $H P(+)$ GC group compared to $H P(+)$ Gastritis group (Fig. 1A). For the expression of RAGE, western blot analysis showed that RAGE expression was increased in $H P(+)$ Gastritis and GC groups compared to $H P$ (-) groups, and remained over-expressed in $H P(+)$ GC group compared to $H P(+)$ Gastritis group (Fig. 1B).

\section{Lowly-expressed miR-1915 and over-expressed RAGE are detected in $H P(+)$ GC cells in vitro}

To examine the in vitro expression of miR-1915 and RAGE during the development of $H P$-associated GC, GES-1, SGC7901, and MKN45 cell lines were obtained and infected with H. pylori at M.O.I of 100:1. Total RNA was extracted $12 \mathrm{~h}$ after $H$. pylori infection. Before the infection, expression of miR1915 was reduced and expression of RAGE was enhanced in $H P(-) 7901$ and MKN45 cells compared to HP (-) GES-1 cells (Supplementary Fig. 2, only online). After H. pylori infection, qRT-PCR results showed that miR-1915 expression was reduced in $H P(+)$ GES-1, 7901, and MKN45 cells compared to their HP (-) controls. Moreover, expression of miR-1915 remained under-expressed in $H P(+) 7901$ and MKN45 cells compared to
$H P(+)$ GES-1 cells (Fig. 2A). In contrast, western blotting results showed that RAGE expression was increased in $H P(+)$ GES-1, 7901, and MKN45 cells compared to their $H P(-)$ controls, and remained over-expressed in $H P(+) 7901$ and MKN45 cells compared to $H P(+)$ GES-1 cells (Fig. 2B). Together, these data demonstrate that miR-1915 is lowly-expressed, but RAGE is over-expressed in $H P(+)$ GC cells in vivo and in vitro.

\section{Over-expressed miR-1915 inhibits proliferation, invasion, and migration of $H P(+)$ GC cells}

Given the lowly-expressed miR-1915 in $H P(+)$ GC cells, we sought to elucidate the causative effect in cell proliferation, invasion, and migration. The $H P(+) 7901$ and MKN45 cells were transfected by miR-1915 mimic, thus the expression of miR1915 was increased (Fig. 3A). Then GC cell proliferating capability was examined by CCK8 assay. We observed the procedures of cellular growth in $0,24,48$, and $72 \mathrm{~h}$, and found that GC cell proliferate capability was attenuated in miR-1915 mimic group compared to pre-NC group (Fig. 3B). GC cell invasion and migration capability were also examined in $H P(+) 7901$ and MKN45 cells by transwell assays, and the results showed a decline in miR-1915 mimic group (Fig. 3C). Taken together, these data demonstrate that up-regulation of miR-1915 inhibits cell proliferation, invasion, and migration of $H P(+)$ GC cells.
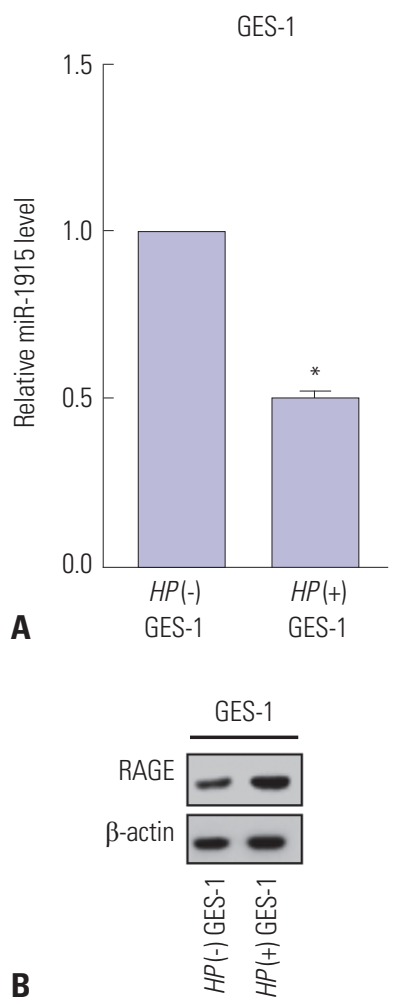
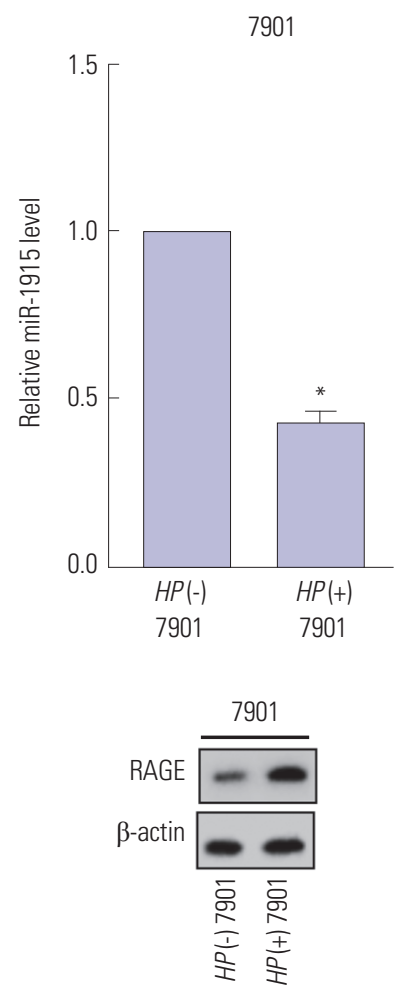
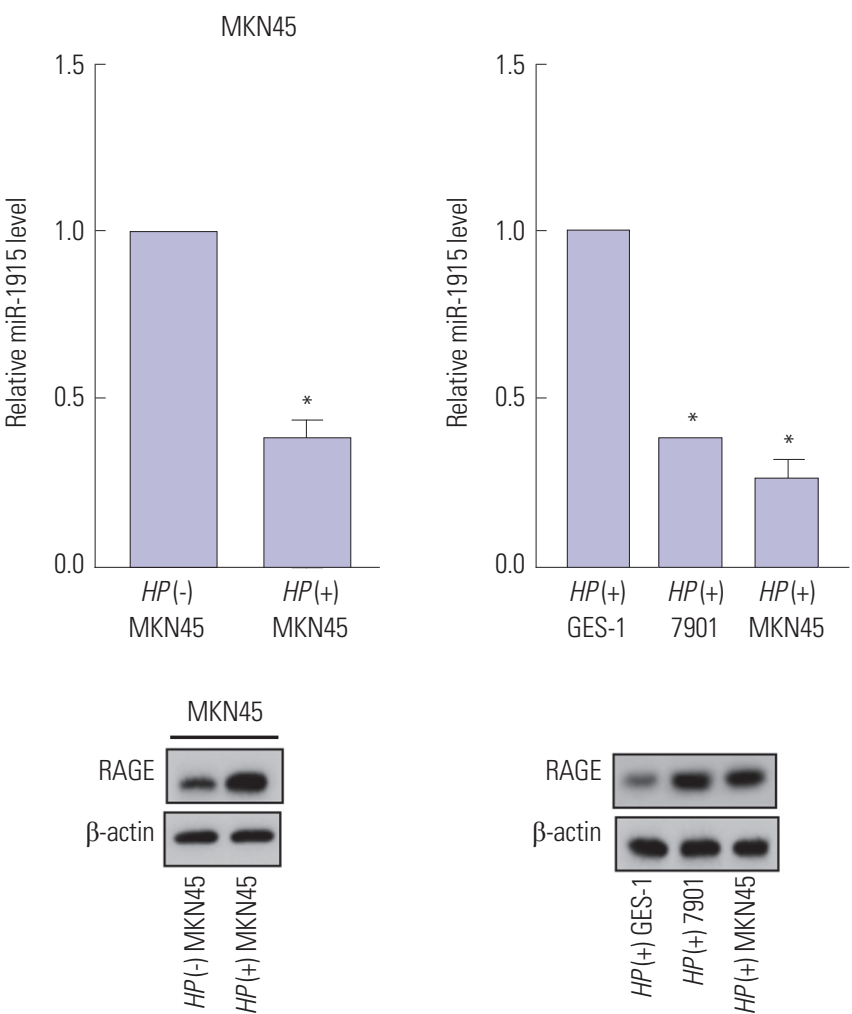

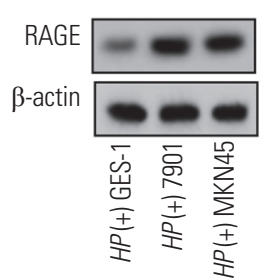

Fig. 2. Under-expressed miR-1915 and over-expressed RAGE in HP(+) GC cells. (A) HPinfection was performed in human SGC-7901, MKN45 GC cell lines, and GES-1 human gastric epithelial cell line. Relative miR-1915 level was detected in each cell line with or without HP infection by qRT-PCR. (B) Expression of RAGE was detected in each cell line with or without $H P$ infection by western blotting. ${ }^{*} p<0.05$ vs. $H P(-)$ GES-1 or $H P(-) 7901$ or $H P(-)$ MKN45 or $H P$ (+) GES-1. RAGE, receptor for advanced glycation end product; HP, Helicobacter pylori. 


\section{There is a target relationship between miR-1915 and RAGE}

To achieve the molecular regulation pattern between miR1915 and RAGE, we introduced miR-1915 inhibitor into 293T cells. Software predicted that miR-1915 could bind to 3'UTR region of RAGE (Fig. 4A). Luciferase reporter assay showed that the relative luciferase activity of RAGE 3'UTR (WT) was significantly increased. Additionally, RAGE mRNA level and RAGE protein expression were also increased, as detected by qRT-PCR and western blot analysis (Fig. 4B). We also introduced miR-1915 mimic into 293T cells, and thus the relative luciferase activity of RAGE 3'UTR (WT) was significantly reduced. The qRT-PCR and western blot analysis results revealed that RAGE mRNA level and RAGE protein expression were both reduced (Fig. 4C). Therfore, we could prove that there is a target relationship between miR-1915 and RAGE.

\section{Molecular mechanism of $H P(+)$ GC cell proliferation,} invasion, and migration via miR-1915

To confirm the specific molecular mechanisms mediated by miR-1915, HP (+) 7901 and MKN45 cells were transfected with
miR-1915 mimic and treated with pcDNA-RAGE, and corresponding control groups were established. The qRT-PCR results showed that miR-1915 level was significantly increased after transfected by miR-1915 mimic compared to pre-NC group, while it remained unchanged after being treated with pcDNA-RAGE compared to pcDNA group (Fig. 5A). On the other hand, RAGE mRNA level and RAGE protein expression were under-expressed after being transfected by miR-1915 mimic, and they remained over-expressed after being treated with pcDNA-RAGE in $H P(+) 7901$ and MKN45 GC cells (Fig. 5B).

Speaking of cell proliferation, invasion, and migration, $H P$ (+) 7901 and MKN45 cells were transfected as previously described, and examined by CCK-8 and transwell assays. The results revealed that cellular capabilities of proliferation, invasion, and migration were all inhibited after transfected by miR1915 mimic, but were promoted after being treated with pcDNARAGE (Fig. 5C, D, and E). Based on these results, we conclude that over-expressed miR-1915 modulation of under-expressed of RAGE may be the mechanism which attenuates the proliferation, invasion, and migration of $H P(+)$ GC cells.
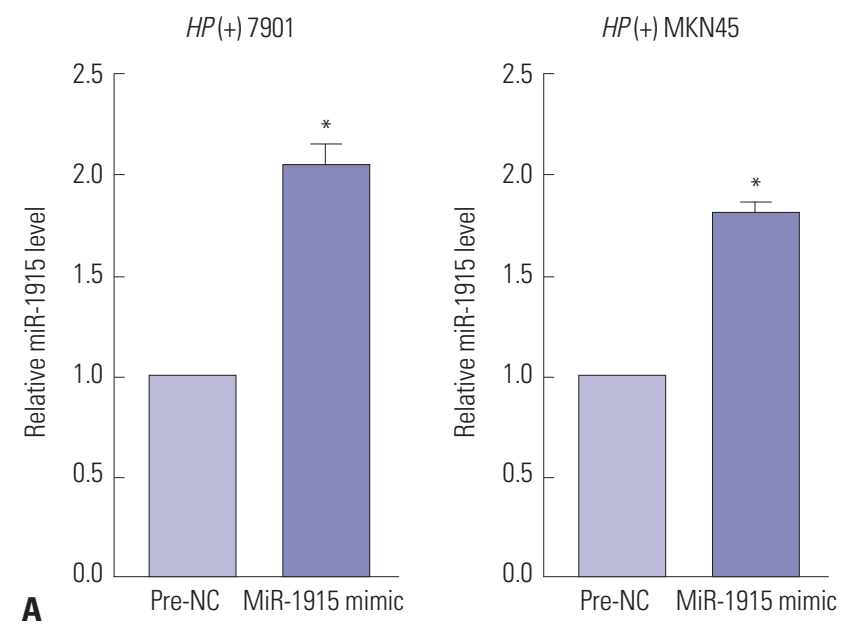
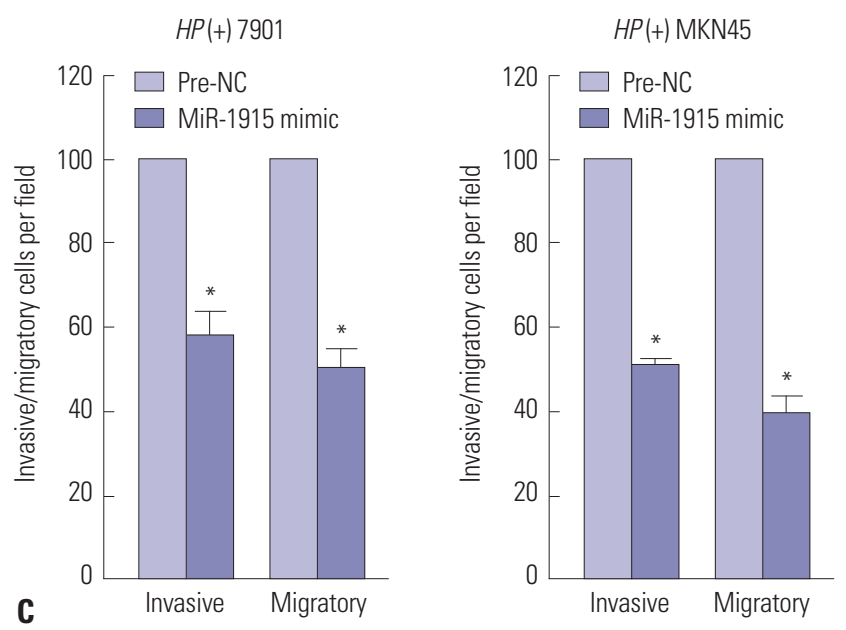
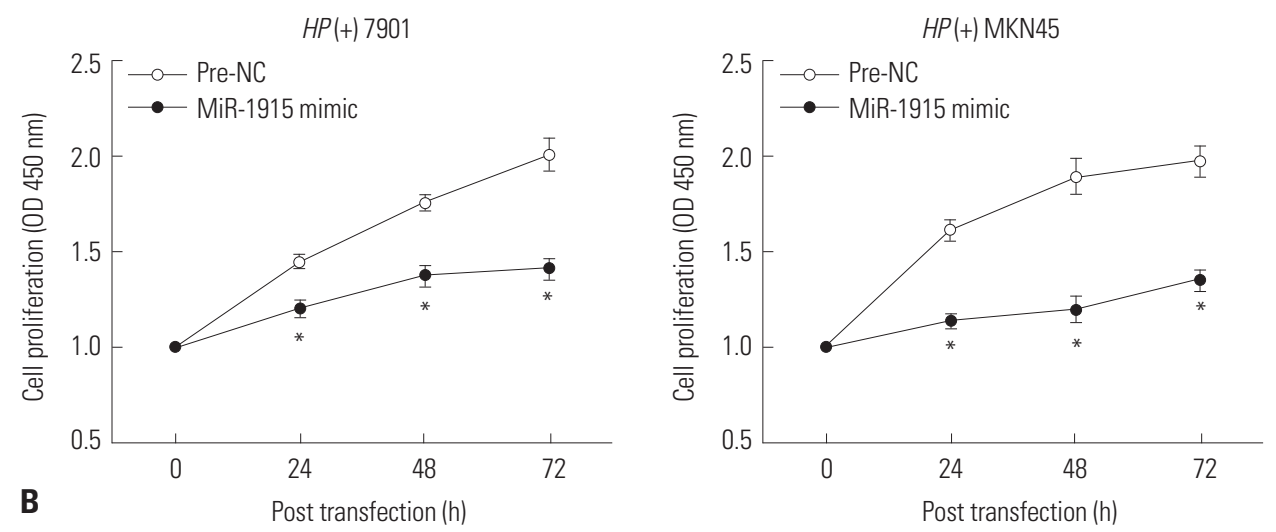

Fig. 3. Over-expressed miR-1915 inhibits proliferation, invasion, and migration of $H P(+) \mathrm{GC}$ cells. $(\mathrm{A}) H P(+) 7901$ and MKN45 cells were transfected by miR-1915 mimic. Relative miR-1915 level was detected by qRT-PCR. (B) Cell proliferation of $H P(+) 7901$ and MKN45 cells in 0, 24, 48, and 72h was detected by CCK8 assay. (C) Invasive/migratory capability of $H P(+) 7901$ and MKN45 cells was detected by transwell assay. ${ }^{*} p<0.05$ vs. pre-NC. GC, gastric cancer; $H P$, Helicobacter pylori. 

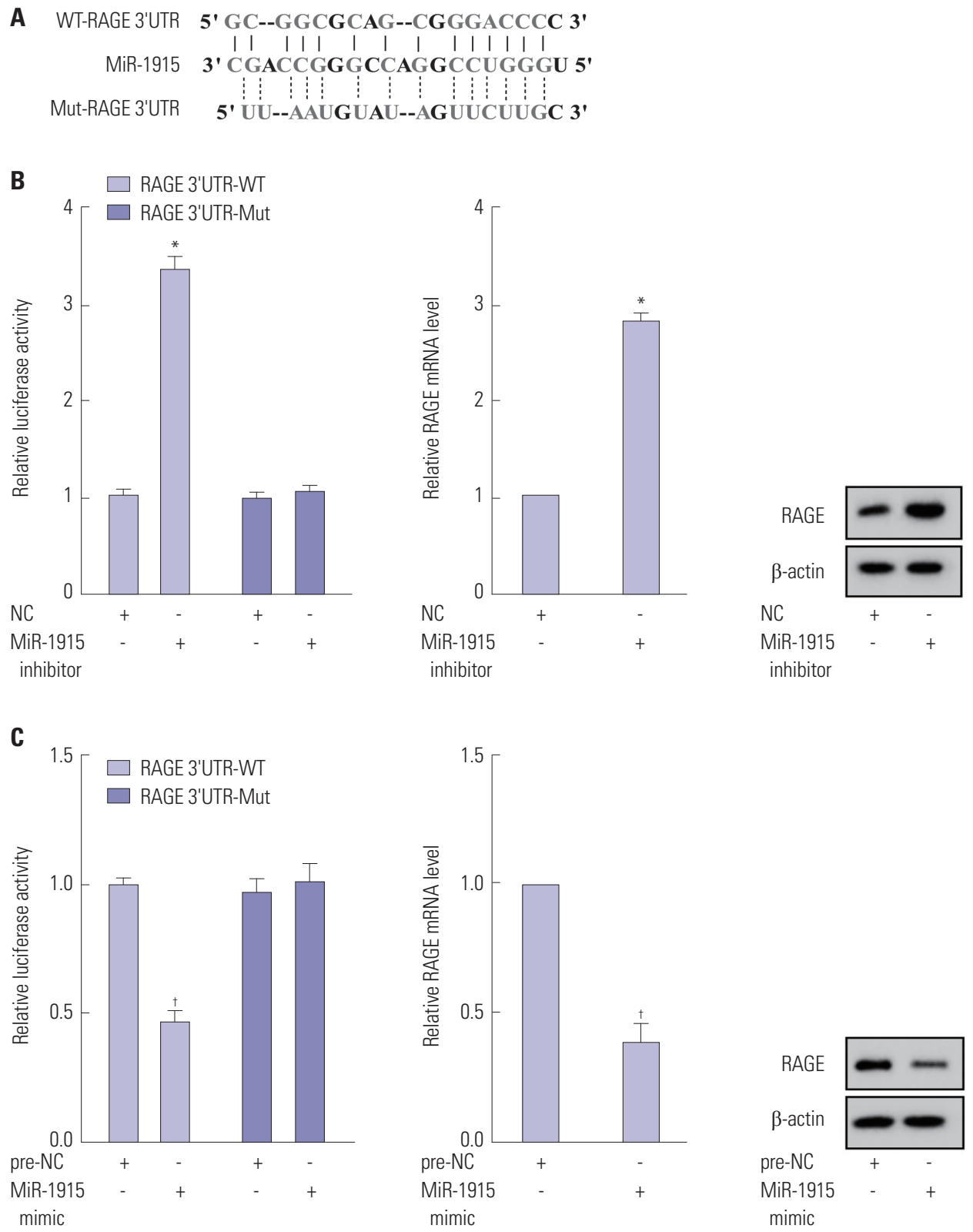

Fig. 4. Target relationship between miR-1915 and RAGE. (A) Complementary sequence information of miR-1915 and RAGE. (B) 293T cells were transfected by miR-1915 inhibitor. Relative luciferase activity of RAGE 3'UTR (WT or Mut) was detected by luciferase reporter assay. Expression of RAGE was detected by qRT-PCR and western blotting. (C) 293T cells were transfected by miR-1915 mimic. Relative luciferase activity of RAGE 3'UTR (WT or Mut) was detected by luciferase reporter assay. Expression of RAGE was detected by qRT-PCR and western blotting. ${ }^{*} p<0.05$ vs. NC, ${ }^{\dagger} p<0.05$ vs. pre-NC. RAGE, receptor for advanced glycation end product; HP, Helicobacter pylori.

\section{DISCUSSION}

GC is a fatal disease, which is also known to be the second most common cause of malignant neoplastic disease-related deaths worldwide, ${ }^{23}$ and it is still difficult to cure this condition. The mechanisms by which $H P$-associated gastritis develops into GC is multifactorial. HP infection induces expression of proinflammatory cytokines, such as interleukin (IL)-8 and tumor necrosis factor (TNF)- $\alpha$, by transactivation of SRE and AP-1 in GC. ${ }^{6} H P$ infection also activates the cyclin D1 gene through mitogen-activated protein kinase (MAPK) pathway and overexpression of cyclin D1 accelerates cell aberrant proliferation. ${ }^{5}$ In addition, activation of telomerase, ras, c-met, $c$ $m y c$, and $c$-erbB-2 genes, as well as the inactivation of $p 53$ gene are involved in $H P$-associated gastric malignant tumorigenesis. ${ }^{9}$ To this end, we can conclude that $H P$ infection significantly increases the incidence of GC, and clarify that the mechanisms between them are particularly important for treatment of GC.

Unlike tumor-suppressor-miRNAs, oncomiRNAs are usually over-expressed in GC, and they promote the proliferation of GC cells, which is a causally step in cancer development. MiR- 
A

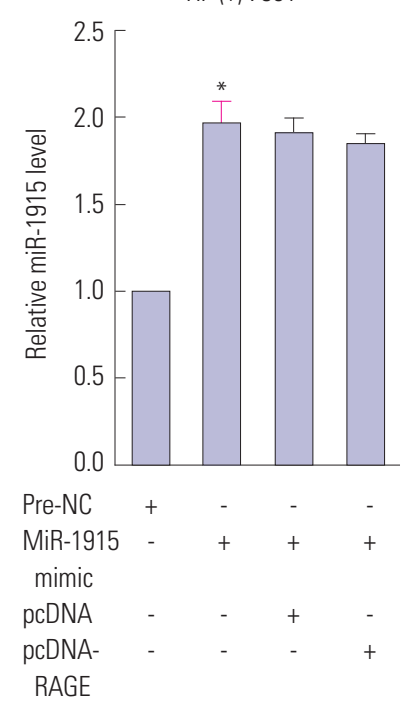

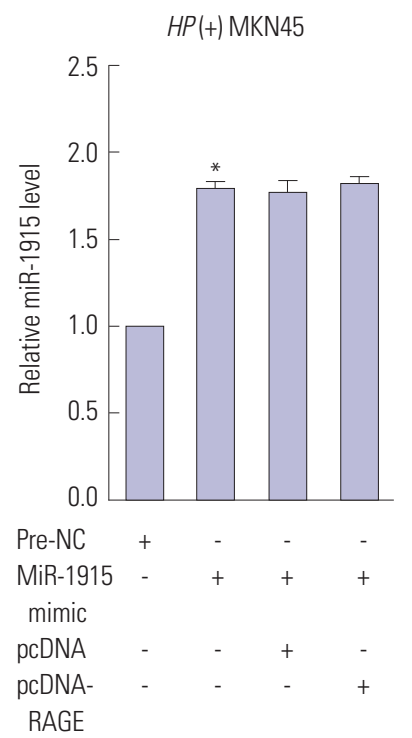

B

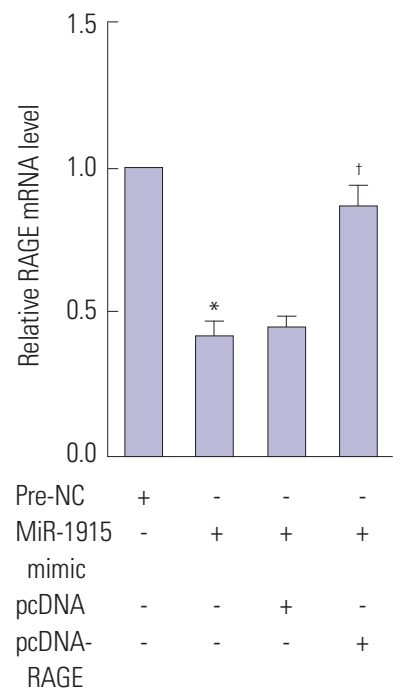

$H P(+)$ MKN45

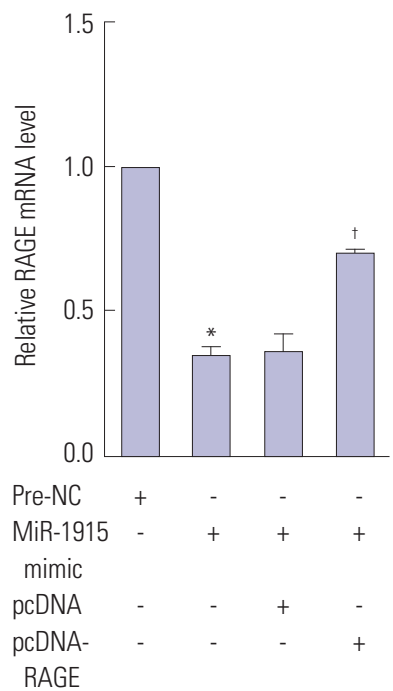

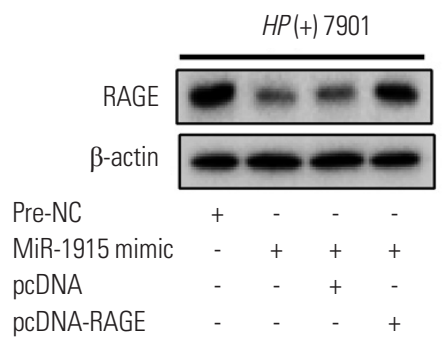

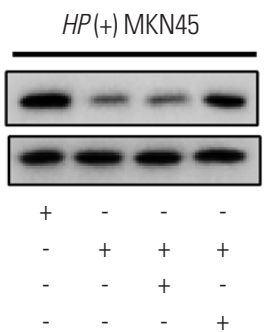

Fig. 5. Proliferation, invasion, and migration of $H P(+)$ GC cells induced by miR-1915 and RAGE. $(A) H P(+) 7901$ and MKN45 cells were transfected by miR1915 mimic, miR-1915 mimic+pcDNA, or miR-1915 mimic+pcDNA-RAGE. Relative miR-1915 level was detected by qRT-PCR. (B) Expression of RAGE was detected by qRT-PCR and western blotting. ${ }^{*} p<0.05$ vs. pre-NC, ${ }^{\dagger} p<0.05$ vs. pcDNA. RAGE, receptor for advanced glycation end product; $H P, H e l i c o b a c t e r$ pylori, GC, gastric cancer.

199a/b-3p, ${ }^{24}$ miR-21, ${ }^{25}$ miR-130b, ${ }^{26}$ and miR23a ${ }^{27}$ were confirmed to be oncomiRNAs promoting GC cell proliferation. However, over-expression of tumor-suppressor-miRNAs leads to the slowdown of cancer cell growth. ${ }^{28}$ Jin, et al. ${ }^{29}$ found that miR-582-5p suppressed GC cell proliferation via targeting AKT3. The expressions of miR-181c, ${ }^{30} \mathrm{miR}-212{ }^{31}$ and $\mathrm{miR}-512^{32}$ were silenced with DNA hypermethylation in GC, and their restored expressions could induce reduced GC cell growth through inhibition of oncogenes expression. Aside from cell proliferation, cell invasion and migration are both important indicators for assessing the malignancy of cancer cells. Ectopic expression of miR-101 has been proven to inhibit cell migration and invasion of GC cells via mediating MCL-1, FOS, EZH2, and COX-2 genes. ${ }^{33}$ Down-regulation of miR-335 was causally related to metastasis of GC lymph-node, as well as invasion of lymphatic vessels. ${ }^{34}$ Although an accumulation of studies on onco-related miRNAs were performed in recent years, most of them focused on GC cells rather than HP-infected GC cells. Our current study confirmed that miR-1915 exerted tumor-suppressive effects on $H P(+)$ GC cell proliferation, invasion, and migration, which provided an innovative and candidate target for treatment of $H P$-infected GC. Before $H$. pylori infection, expression of miR-1915 was reduced while expression of RAGE was enhanced in $H P(-) 7901$ and MKN45 cells compared to $H P(-)$ GES-1 cells (Supplementary Fig. 2, only online). Combined with the down-regulation of miR-1915 in HP (-) GC tissues, these data indicated that miR-1915 was down-regulated in both $H P(-)$ GC tissues and cells; therefore, their underlying mechanisms deserve further investigation in our future research.

Recent studies have confirmed that miR-1915 is related to carcinogenesis. For instance, miR-1915-3p has been identified as oxidative stress-responsive miRNA regulated by p53-dependent pathway in antiapoptosis process in hepatocellular cancer. ${ }^{9}$ Such antiapoptosis function of miR-1915-3p has also been identified in lung cancer by targeting GTP-binding protein 2 (DRG2)/pre-B cell leukemia homeobox 2 (PBX2). ${ }^{10}$ Furthermore, miR-1915-3p enhances breast cancer cell proliferative and migrational abilities by targeting gene DUSP3 and activating ERK1/2. ${ }^{11}$ On the contrary, our work revealed the negative effect of miR-1915 in cell proliferation, invasion, and migration of $H P$-infected GC cells by targeting RAGE. We assumed that this may be associated with the infection of $H P$ and immunologic function of RAGE. Taken together, these data have revealed that miR-1915 functions as either oncogenes or tumor suppressor genes depending on the roles of its target gene, 

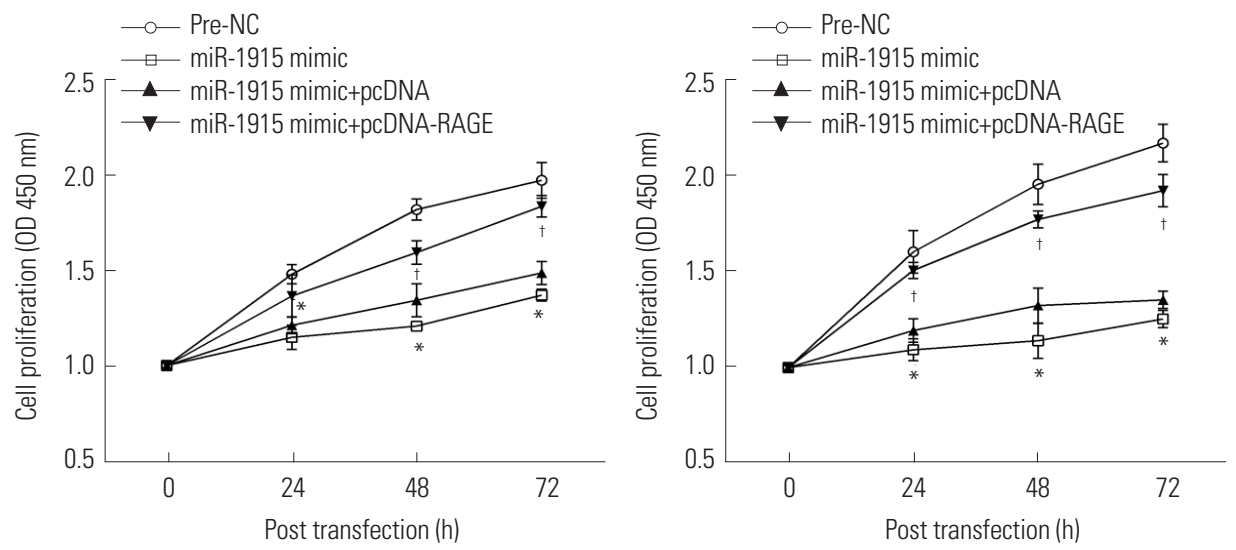

D

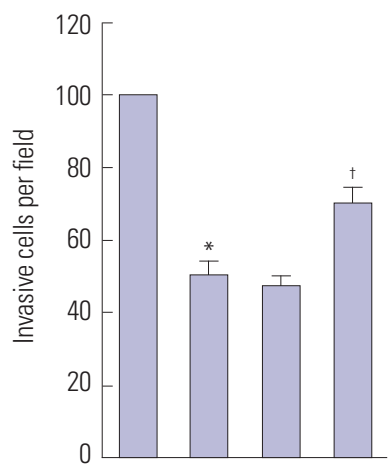

Pre-NC

MiR-1915 - + + +

mimic

pcDNA

pcDNA-

RAGE

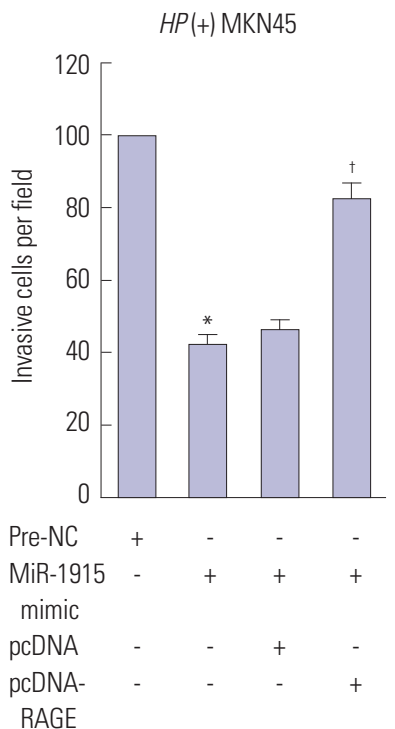

E

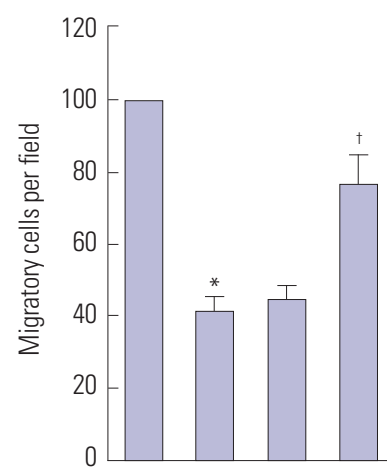

Pre-NC

MiR-1915

mimic

pcDNA

pcDNA-

RAGE

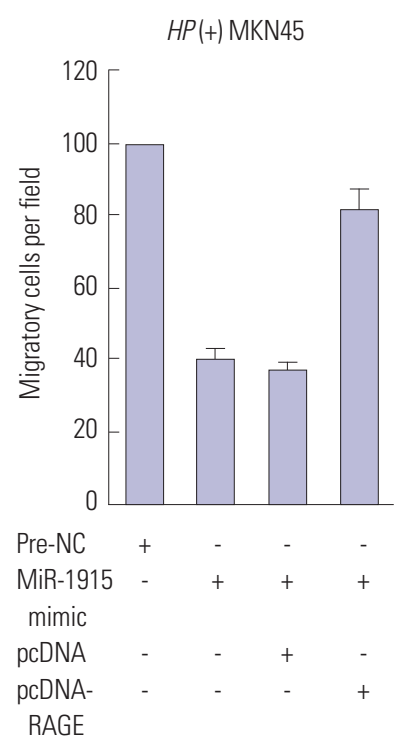

Fig. 5. Proliferation, invasion, and migration of $H P(+)$ GC cells induced by miR-1915 and RAGE. (C) Cell proliferation in $0,24,48$, and $72 \mathrm{~h}$ was detected by CCK-8 assay. (D) Cell invasive capability was detected by transwell assay. (E) Cell migratory capability was detected by transwell assay. ${ }^{*} p<0.05$ vs. pre$\mathrm{NC},{ }^{\dagger} p<0.05$ vs. pcDNA. RAGE, receptor for advanced glycation end product; $H P$, Helicobacter pylori, GC, gastric cancer.

which emphasizes the importance of miR-1915 in different cancer treatments.

The above mentioned mechanism needs an important molecule participation-RAGE. It is a pattern recognition receptor which also plays an important role in the occurrence of GC via binding multiple ligands derived from a damaged cell environment. ${ }^{35}$ RAGE has previously been shown to be related to invasion and metastasis in $\mathrm{GC},{ }^{36}$ which indicates a research base for the current study of RAGE in GC. To this end, our former study focused on RAGE in GC, and proved that knockdown of RAGE could inhibit growth and invasion of GC cells. ${ }^{15}$ Inspired by our former research, we assumed that targeted inhibition of RAGE may act as novel targets to improve current cancer therapies. In this study, we detected RAGE expression in $H P$ infected GC tissues and cells by using several systematic and bioinformatic approaches, and first found that RAGE expression could be reduced by up-regulating miR-1915 by which cellular proliferation, invasion, and migration were inhibited in
$H P$-infected GC cells. Similarly, the role of RAGE in other cancer types, by promoting cell proliferation and tumor metastases, has been reported. For instance, inhibition of RAGE decreased cell proliferation of breast cancer cells, ${ }^{37}$ induced cell apoptosis, and inhibited growth of prostate cancer. ${ }^{38}$ RAGE dysfunction inhibited colorectal cancer angiogenesis and its progression as well, ${ }^{39}$ and prolonged the survival rate of patients who were diagnosed with pancreatic cancer. ${ }^{40}$ In addition to the relationship between miR-1915 and various types of cancers, miR-1915/RAGE pathway in other types of malignant cells deserves further investigation.

In conclusion, we confirmed that miR-1915 exerted tumorsuppressive effects on $H P$-infected GC cellular proliferation, invasion, and migration via targeting RAGE, which may be a novel prognostic biomarker and a potential therapeutic target for $H P$ infected GC patients. 


\section{ACKNOWLEDGEMENTS}

This study was supported by 2017 Natural Science Fund Project of Autonomous Region (2017D01C305).

\section{ORCID iDs}

$\begin{array}{ll}\text { Xin-cai Xu } & \text { https://orcid.org/0000-0001-6087-0380 } \\ \text { Wen-bin Zhang } & \text { https://orcid.org/0000-0003-3398-6331 } \\ \text { Chun-xing Li } & \text { https://orcid.org/0000-0001-8090-5165 } \\ \text { Hua Gao } & \text { https://orcid.org/0000-0001-8869-2213 } \\ \text { Qi Pei } & \text { https://orcid.org/0000-0002-8864-6214 } \\ \text { Bo-wei Cao } & \text { https://orcid.org/0000-0003-4464-9509 } \\ \text { Tie-han He } & \text { https://orcid.org/0000-0003-4653-6276 }\end{array}$

\section{REFERENCES}

1. Mbulaiteye SM, Hisada M, El-Omar EM. Helicobacter Pylori associated global gastric cancer burden. Front Biosci (Landmark Ed) 2009;14:1490-504.

2. Howson CP, Hiyama T, Wynder EL. The decline in gastric cancer: epidemiology of an unplanned triumph. Epidemiol Rev 1986;8:1-27.

3. Talley NJ, Zinsmeister AR, Weaver A, DiMagno EP, Carpenter HA, Perez-Perez GI, et al. Gastric adenocarcinoma and Helicobacter pylori infection. J Natl Cancer Inst 1991;83:1734-9.

4. Parkin DM. The global health burden of infection-associated cancers in the year 2002. Int J Cancer 2006;118:3030-44.

5. Hirata Y, Maeda S, Mitsuno Y, Akanuma M, Yamaji Y, Ogura K, et al. Helicobacter pylori activates the cyclin D1 gene through mitogenactivated protein kinase pathway in gastric cancer cells. Infect Immun 2001;69:3965-71.

6. Mitsuno Y, Yoshida H, Maeda S, Ogura K, Hirata Y, Kawabe T, et al. Helicobacter pylori induced transactivation of SRE and AP-1 through the ERK signalling pathway in gastric cancer cells. Gut 2001;49:18-22.

7. Wang J, Yu JC, Kang WM, Ma ZQ. Treatment strategy for early gastric cancer. Surg Oncol 2012;21:119-23.

8. Shin VY, Chu KM. MiRNA as potential biomarkers and therapeutic targets for gastric cancer. World J Gastroenterol 2014;20:10432-9.

9. Wan Y, Cui R, Gu J, Zhang X, Xiang X, Liu C, et al. Identification of four oxidative stress-responsive microRNAs, miR-34a-5p, miR1915-3p, miR-638, and miR-150-3p, in hepatocellular carcinoma. Oxid Med Cell Longev 2017;2017:5189138.

10. Xu C, Li H, Zhang L, Jia T, Duan L, Lu C. MicroRNA19153p prevents the apoptosis of lung cancer cells by downregulating DRG2 and PBX2. Mol Med Rep 2016;13:505-12.

11. Guo J, Liu C, Wang W, Liu Y, He H, Chen C, et al. Identification of serum miR-1915-3p and miR-455-3p as biomarkers for breast cancer. PLoS One 2018;13:e0200716.

12. Xu K, Liang X, Cui D, Wu Y, Shi W, Liu J. miR-1915 inhibits Bcl-2 to modulate multidrug resistance by increasing drug-sensitivity in human colorectal carcinoma cells. Mol Carcinog 2013;52:70-8.

13. Zhang HH, Gu GL, Zhang XY, Li FZ, Ding L, Fan Q, et al. Primary analysis and screening of microRNAs in gastric cancer side population cells. World J Gastroenterol 2015;21:3519-26.

14. Wang D, Li T, Ye G, Shen Z, Hu Y, Mou T, et al. Overexpression of the receptor for advanced glycation endproducts (RAGE) is associated with poor prognosis in gastric cancer. PLoS One 2015;10: e0122697.

15. Xu XC, Abuduhadeer X, Zhang WB, Li T, Gao H, Wang YH. Knockdown of RAGE inhibits growth and invasion of gastric can- cer cells. Eur J Histochem 2013;57:e36.

16. Xu X, Cao B, Zhang Y, He T, Li T, Zhang W. Mechanism of synergistic effect of Helicobacter pylori and RAGE expression on gastric cancer. Chi J Gastrointest Surg 2017;20:1072-4.

17. Zhou X, Chen H, Zhu L, Hao B, Zhang W, Hua J, et al. Helicobacter pylori infection related long noncoding RNA (lncRNA) AF147447 inhibits gastric cancer proliferation and invasion by targeting MUC2 and up-regulating miR-34c. Oncotarget 2016;7:82770-82.

18. Ma L, Chen Y, Zhang B, Liu G. Increased microRNA-223 in Helicobacter pylori-associated gastric cancer contributed to cancer cell proliferation and migration. Biosci Biotechnol Biochem 2014;78: 602-8.

19. Xue TM, Tao LD, Zhang M, Xu GC, Zhang J, Zhang PJ. miR-20b overexpression is predictive of poor prognosis in gastric cancer. Onco Targets Ther 2015;8:1871-6.

20. Liu W, Kang L, Han J, Wang Y, Shen C, Yan Z, et al. miR-342-3p suppresses hepatocellular carcinoma proliferation through inhibition of IGF-1R-mediated Warburg effect. Onco Targets Ther 2018; 11:1643-53.

21. Yu Z, Zhang W, Deng F. MicroRNA-577 inhibits gastric cancer growth by targeting E2F transcription factor 3. Oncol Lett 2015; 10:1447-52.

22. Kim CH, Kim HK, Rettig RL, Kim J, Lee ET, Aprelikova O, et al. miRNA signature associated with outcome of gastric cancer patients following chemotherapy. BMC Med Genomics 2011;4:79.

23. Jemal A, Siegel R, Xu J, Ward E. Cancer statistics, 2010. CA Cancer J Clin 2010;60:277-300.

24. Zeng B, Shi W, Tan G. MiR-199a/b-3p inhibits gastric cancer cell proliferation via down-regulating PAK4/MEK/ERK signaling pathway. BMC Cancer 2018;18:34.

25. Zhang Z, Li Z, Gao C, Chen P, Chen J, Liu W, et al. miR-21 plays a pivotal role in gastric cancer pathogenesis and progression. Lab Invest 2008;88:1358-66.

26. Lai KW, Koh KX, Loh M, Tada K, Subramaniam MM, Lim XY, et al. MicroRNA-130b regulates the tumour suppressor RUNX3 in gastric cancer. Eur J Cancer 2010;46:1456-63.

27. Zhu LH, Liu T, Tang H, Tian RQ, Su C, Liu M, et al. MicroRNA-23a promotes the growth of gastric adenocarcinoma cell line MGC803 and downregulates interleukin-6 receptor. FEBS J 2010;277:3726-34.

28. Wu HH, Lin WC, Tsai KW. Advances in molecular biomarkers for gastric cancer: miRNAs as emerging novel cancer markers. Expert Rev Mol Med 2014;16:e1.

29. Jin Y, Tao LP, Yao SC, Huang QK, Chen ZF, Sun YJ, et al. MicroRNA-582-5p suppressed gastric cancer cell proliferation via targeting AKT3. Eur Rev Med Pharmacol Sci 2017;21:5112-20.

30. Hashimoto Y, Akiyama Y, Otsubo T, Shimada S, Yuasa Y. Involvement of epigenetically silenced microRNA-181c in gastric carcinogenesis. Carcinogenesis 2010;31:777-84.

31. Wada R, Akiyama Y, Hashimoto Y, Fukamachi H, Yuasa Y. miR-212 is downregulated and suppresses methyl-CpG-binding protein MeCP2 in human gastric cancer. Int J Cancer 2010;127:1106-14.

32. Saito Y, Suzuki H, Tsugawa H, Nakagawa I, Matsuzaki J, Kanai Y, et al. Chromatin remodeling at Alu repeats by epigenetic treatment activates silenced microRNA-512-5p with downregulation of Mcl1 in human gastric cancer cells. Oncogene 2009;28:2738-44.

33. He XP, Shao Y, Li XL, Xu W, Chen GS, Sun HH, et al. Downregulation of miR-101 in gastric cancer correlates with cyclooxygenase-2 overexpression and tumor growth. FEBS J 2012;279:4201-12.

34. Xu Y, Zhao F, Wang Z, Song Y, Luo Y, Zhang X, et al. MicroRNA-335 acts as a metastasis suppressor in gastric cancer by targeting Bcl$\mathrm{w}$ and specificity protein 1. Oncogene 2012;31:1398-407.

35. Heijmans J, Büller NV, Hoff E, Dihal AA, van der Poll T, van Zoelen MA, et al. Rage signalling promotes intestinal tumourigenesis. 
Oncogene 2013;32:1202-6.

36. Kuniyasu H, Oue N, Wakikawa A, Shigeishi H, Matsutani N, Kuraoka K, et al. Expression of receptors for advanced glycation endproducts (RAGE) is closely associated with the invasive and metastatic activity of gastric cancer. J Pathol 2002;196:163-70.

37. Radia AM, Yaser AM, Ma X, Zhang J, Yang C, Dong Q, et al. Specific siRNA targeting receptor for advanced glycation end products (RAGE) decreases proliferation in human breast cancer cell lines. Int J Mol Sci 2013;14:7959-78.

38. Elangovan I, Thirugnanam S, Chen A, Zheng G, Bosland MC, Kaj-
dacsy-Balla A, et al. Targeting receptor for advanced glycation end products (RAGE) expression induces apoptosis and inhibits prostate tumor growth. Biochem Biophys Res Commun 2012;417:1133-8.

39. Liang H, Zhong Y, Zhou S, Peng L. Knockdown of RAGE expression inhibits colorectal cancer cell invasion and suppresses angiogenesis in vitro and in vivo. Cancer Lett 2011;313:91-8.

40. DiNorcia J, Lee MK, Moroziewicz DN, Winner M, Suman P, Bao F, et al. RAGE gene deletion inhibits the development and progression of ductal neoplasia and prolongs survival in a murine model of pancreatic cancer. J Gastrointest Surg 2012;16:104-12. 